\title{
Detection and Tracking of Lane Crossing Vehicles in Traffic Video for Abnormality Analysis
}

\author{
Arun Kumar H D, Prabhakar C J
}

\begin{abstract}
In this paper, we present a novel approach for detection and tracking of lane crossing/illegal lane crossing vehicles in traffic video of urban highways. For that intention, an initial pace is performed that estimates the road region of the geometrical structure. After finding the road region, every vehicle is tracked in order to detect lane crossing vehicles according to the distance between lane lines and vehicle centre, it is followed by tracking of lane crossing vehicles based on model-based strategy. The proposed system has been evaluated using recall and precision metric, which are received using experiments carried on selected video sequences of GRAM-RTM dataset and publically available video sequences. The experimental results present that our method reaches the highest accuracy for detection of vehicles and tracking of lane crossing vehicles.
\end{abstract}

Keywords: Illegal Lane Crossing, Abnormal Events, Lane Line Detection, Background Detection

\section{INTRODUCTION}

Robust traffic supervising and surveillance have become necessary for road usage and direction. Different sensors or components are used to evaluation vehicles traffic parameters. Magnetic loop detectors have been the most normally used sensors, but they are costly and difficult to install and maintain. Compared to magnetic loop detectors, video sensors become especially significant in traffic monitoring and surveillance mainly due to their easy installation, fast reaction, operation, and maintenance, and their power to monitor broad areas. More and more video surveillance cameras are installed on heavy traffic roads, highways and urban intersection areas. However, they are not optimally used due to the manual watching of the output, which is costly and unreliable. It is clear that manual supervising of traffic issues from various different screens expect high focus and special density of the traffic operators, and there are always theories to miss the urgent events in the manual watching scene. Robotic surveillance systems aim to incorporate real-time and effective computer vision algorithms in order to attend to human operators. This is challenging goal which has attracted an enhancing amount of researchers to solve normally come across video surveillance

Manuscript received on January 05, 2021.

Revised Manuscript received on March 30, 2021.

Manuscript published on April 30, 2021.

* Correspondence Author

Arun Kumar H D*, Lecturer, Department of Computer Science and MCA, Kuvempu University, Karnataka, India.

Prabhakar C J, Associate Professor, Department of Computer Science and M.C.A, Kuvempu University, Karnataka, India.

(C) The Authors. Published by Blue Eyes Intelligence Engineering and Sciences Publication (BEIESP). This is an open access article under the CC BY-NC-ND license (http://creativecommons.org/licenses/by-nc-nd/4.0/)

problems of vehicle classification and vehicle abnormality we centered on detection and tracking of lane crossing/illegal lane crossing vehicles which is one of the important abnormal events in highways and intersection in the urban area. The lanal lane crossing is an abnormal event where the vehicle challenging with observe to vehicles density, lower camera angles so as to take to a high degree of occlusion, and the form road vehicle users.

[1] authors have proposed a method to detect lane for creation of background modeling. They use a Sobel operator and lane markings are extract using the Hough transformation. To detect moving foreground vehicles, they apply background-frame differencing. Once a vehicle is detected (noticed), the center of the detected moving blob is used to verify whether the detected vehicle is changing lanes illegally. In [2] authors have proposed a system for detection of illegal crossings of solid lines using computer vision and image processing techniques on video or image sequences captured from IP surveillance cameras. A background model is used to detect the foreground from the background in the image. The proposed system focuses on detecting moving cles blobs successfully and uses the intersection of the base profile of the blob with the violation region in order to discover violations.

In [3] authors have proposed monitoring system to detect lane crossing vehicles based on vehicle tracking information. method failed to detect lane crossing vehicles when the vehicle is too close to the lane line and multiple vehicles are connected by their shadows because they used mean-shift tracker and Kalman filters to track the moving vehicles. It is erved that the mean shift tracker failed to track the vehicles when the vehicle changes their pose, directions and when vehicle occlusion occurs. In order to overcome these limitations, we used our previous work [4] for tracking vehicles which is a model-based technique. In the del-based approach, there are two key components: vehicle demonstration and dynamics. Vehicle demonstration tests to model the vehicle as right as potential so that the tracking algorithm can correctly label the difficult vehicle appearance.

The vehicle dynamics model constitutes how the vehicle variations. These two problems are usually matched together. The vehicle demonstration should be designed to simply modify the model based on appearance variations, 


\section{Detection and Tracking of Lane Crossing Vehicles in Traffic Video for Abnormality Analysis}

while the vehicle dynamics should be able to take advantage of the characteristics of vehicle demonstration for model update. We construct a vehicle model which catches the variation in scale of the vehicle, pose of the vehicle, and complex vehicle occlusion based on spatial local features such as shape features are extracted from Co-HOG feature and texture features extracted from CS-LBP operator.

The proposed work aimed to detect and track the lane crossing/illegal lane crossing vehicles in roads using video sequence of traffic scenario captured through fixed a camera. The proposed method involves two steps. In the first step, the background is detected using a Gaussian Mixture Model (GMM) method which helps to find lane region (region of interest). The Hough transformation is used to locate the boundary of the lane using an edge map of the background image. In the second phase, every vehicle is tracked using our previous work [4] which uses model-based tracking technique. While tracking every vehicle, we observe the tracking window and vehicle is said to be lane crossing or illegal overtaking vehicle when it's tracking window center lies the outer boundary of the lane. After illegal lane crossing vehicles are detected, illegal lane crossing vehicles are tracked to expect the location of the illegal vehicle. We carried out experiments using some of the traffic video sequences selected from GRAM-Road Traffic Monitoring dataset (GRAM-RTM) [5]. In addition to GRAM-RTM dataset, we used traffic video sequences available on the Internet and these video sequences contain lane crossing/illegal lane crossing events. The proposed method is evaluated using standard metrics such as precision and recall.

\section{REVIEW CRITERIA}

In the state-of-art, the last two decades, a number of approaches have been proposed for vehicles abnormality detection in highway and intersection area in urban. There are two categories to anomalous event detection depending on the features extracted. First category techniques are based on low-level pixel features, while the second category techniques are based on the high-level features extracted from detection and tracking of the target. Low-level features are extracted using the following techniques: background subtraction, optical flow. Events and action are observed based on the motion patterns from these low-level features. However, in these approaches, the individual objects are not identified and therefore detected anomalies cannot be matched to any particular objects. The second category approaches are the most important and popular approaches based on vehicle tracking and trajectories techniques. A normally used method, in this instance, is based on the clustering the trajectories of the moving objects [6]. Then received clusters are used as vehicle abnormality model for vehicle abnormality detection.

In [7] authors have proposed an approach to report traffic accident events at road intersections in an urban area in a human-understandable way using automated video processing or computer vision techniques. The author proposed approach depends upon training normal traffic flow by trajectory clustering technique such as Continuous Hidden Markov Model (CHMM), in which clustered patterns indicate regular activity paths. Later intersection modeling is finished; new vehicle trajectories are observed continuously. If the model catches a likely accident scene, it informs this event to a traffic controller for high-level human perception. In [8] authors have proposed an image object tracking system and its applications for accident detection and traffic monitoring at road junctions. Positions of vehicles are obtained using active shape model method. Kalman filters technique to track separate vehicle motion. In [9] authors have proposed a semantic hierarchy algorithm for 1) logical reasoning to identify individual behaviour 2) logical reasoning focusing on relative behaviour 3) classification of continuous viable. These three classes are used for detecting anomalous events in traffic video surveillance.

In [10] authors have proposed an abnormal event detection method using switching Kalman filter and combination with some simple rules. They use multiple cues and multiple motion tracking models and event detection such as turning, stopped or stalling vehicles, as well as motion statistics (average speed and acceleration). Based on some simple rules the abnormal actions are differentiated from normal events. The weakness of this proposed work is wrong target estimation and the threshold value. In [11] authors have proposed a method for abnormal event detection using trajectory sequence. This approach is based on single-class SVM clustering. SVM capabilities are used to recognize abnormal events. Trajectory classification in absence of a priori information and the method was used to classify the anomalous events from formula events. The [12] have proposed a vision-based method for automatic detection of traffic event detection. Cellular model is formed by extracting entropy-based features that simulate the dynamic behaviour of the traffic flow. If abnormal events are happening on the vehicular lane, the actions are discovered instantly.

In [13] authors have proposed an algorithm is automatic incidental detection using a support vector machine (SVM). The SVM is trained using incidental point velocity, the downriver velocity, the upriver velocity, the upriver occupancy rate, the incidental point occupancy rate and the downriver occupancy rate. The incidentals are detected using the output of SVM. In [14] authors have proposed an algorithm for automatic abnormal traffic event detection. They used directional motion actions descriptor to assemble moving object's instruction and speed information from the image sequences with the formula traffic events. In the detection phase, the directional motion behaviour is extracted from incoming video sequences and compared with the normal activity descriptor. In [15] authors have proposed a method for abnormal event detection based on local characteristics for video surveillance. The foreground is detected first and area of the foreground region, shape elements (i.e. ellipse eccentricity, the height-width radius of outside rectangular) and velocity of the traveling pixel vector is extracted.

In [16] the author has proposed an unsupervised abnormality event detection method using a distributed video surveillance system at an intersection.

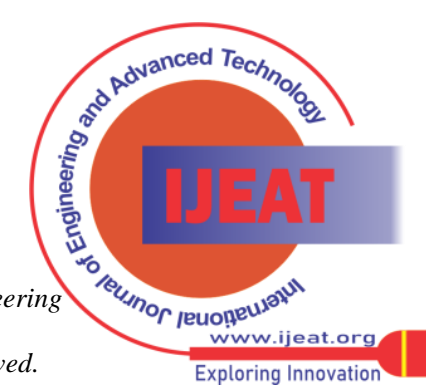


The author analyses activities at an intersection in the number of traffic regions for detecting and classifying traffic vehicles and then tracking to extract traffic flows which support in abnormality detection. The proposed work covers abnormality detection using vehicles trajectory analysis based on support vector machine (SVM). In [17] authors have projected an online trajectory clustering method for vehicle abnormal event detection. The extracted trajectories are clustered and clusters are organized online in a tree structure. The model is capable to update clusters as new trajectories are observed. In [1] authors have suggested a similarity-based vehicle trajectory clustering and abnormality detection. They planned a two-layer hierarchical trajectory clustering algorithm in order that detect common activity actions. Using incomplete vehicle trajectories, possible collision points and collision probabilities are predicted.

\section{DETECTION OF THE ROAD REGION}

In traffic video, according to road traffic rules the vehicles are moving only in a physical scene and geometry of the road area. The other parts of the video scene like a houses, sky, and land areas are not useful for analysis of video traffic and can be ignored. The region of interest is therefore restricted to the road areas. In this section, we present a proposed method for detection of the road region. It can be therefore identified in an early offline learning phase and will be used in the online learning phase to detect illegal lane crossing event.

In order to find the road region, we extract the background of given traffic video using Gaussian Mixture Model [19] technique based on background modeling. The simplest way to the background model is to obtain a background image which doesn't contain any moving element. The background model information is obtained using a Gaussian mixture model. It consists of a Gaussian density of its weighted sum, which allows the color distribution for each pixel to be multi-modal of Gaussian mixture. Modeling the history of pixel values by several normal distributions helps the scheme to be more robust against occlusion and local illumination changes. Typically, $\mathrm{k}=3$ Gaussian distributions are used, the distributions are manually labeled in a heuristic method as follows: the darkest element is label as a shadow; in the remaining two element, the one with the largest variance is labeled as moving vehicle and the other one as road and the parametric quantity of each Gaussian (weight $\omega$, mean $\mu$, and covariance $\Sigma$ ) are modified dynamically throughout time. The likelihood $P$ of occurrence of a color $u$ at present pixel i and time $t$ is given as:

$$
P\left(X_{t}=u\right)=\sum_{i=1}^{k}\left(\omega_{i, t}, \eta\left(X_{t}, \mu_{i, t}, \sum_{i, t}\right)\right),
$$

$$
\eta\left(X_{t}, \mu_{i, t}, \sum_{i, t}\right) \text { is the } \mathrm{i}^{\text {th }} \text { Gaussian distribution and the }
$$
weight is $\omega_{i, t}$. The covariance $\sum_{i, t}$ is because to be diagonal with ${ }^{2}{ }_{i, t}$ as its diagonal elements. For each one pixel, the first step consists of finding the closest equivalent Gaussian distribution, i.e. the Gaussian distribution for which the intensity of the pixel is within $T_{\sigma}$ variation of its mean. The parametric quantity of the matched component are then modified by

$$
\begin{aligned}
\omega_{i, t+1}=(1-\alpha) & \omega_{i, t}+\alpha, \\
\mu_{i, t+1}= & (1-\rho) \mu_{i, t}+\rho X_{t+1}, \\
\sigma_{i, t+1}^{2}= & (1-\rho) \sigma_{i, t}^{2}+\rho\left(X_{t+1}-\mu_{i, t+1}\right),
\end{aligned}
$$

with $\alpha$ being a user-defined learning rate, and $\rho$ defined by

$$
\rho=\alpha \cdot \eta\left(X_{t+1}, \mu_{i, t}, \Sigma_{i, t}\right) .
$$

The weight of matchless components is modified with

$$
\omega_{i, t+1}=(1-\alpha) \omega_{i, t},
$$

and $\mu_{i, t+1}, \sigma_{i, t+1}^{2}$ remain matchless. If no element matched, a new Gaussian distribution with mean $X_{t}$, a prominent variance $\sigma_{0}^{2}$ and a little weight $\omega_{0}$ is created to replace the being Gaussian distribution with the little weight. Once Gaussian distribution are modified based on the value $\omega_{i, t+1} /$

$\sigma_{i, t+1}^{2}$ only the $B$ most reliable distributions are chosen to make up the background, with

$$
B=\underset{k}{\operatorname{argmin}} \sum_{i=1}^{n}\left(\omega_{i, t+1}<T_{b g}\right) .
$$

Pixels which are at more $T_{\alpha}$ standard deviations away from any of those $B$ distributions are marked as foreground image. Figure 1(a) shows the background of the traffic video.

After obtaining background of the traffic video, Canny Edge Detector is used to get edge map of the background image, Figure 1(b) shows that result of the Canny edge detector [19]. When the edge map is identified, straight line parametric quantities are calculated using Standard Hough Transform (SHT) [20]. Figure 1(c) shows the straight lines obtained using SHT. Once the Hough space is computed, local maxima are extracted, and lines that are away from a vanishing point and too short are discarded. This information is used to define the lane on which the vehicles are moving and to detect the vehicles that change the line of traffic. For a vehicle, changing its lane could be permitted or not depending on the traffic rules. Lines that contain continuous edges are considered to be continuous lines elsewhere, they are considered to be broken lines.

\section{A. Vanishing Point (appearance) Estimation}

Theoretically, everyone boundary edge lanes meet to the Vanishing point (VP) or appearance. It is, therefore, interesting to detect it in order to delete or validate the previously estimated boundary edge lines. The Vanishing point is calculated using the [21] approach where the polar space of Hough Transform is exploited. A least square method based on the minimization of the following function is used:

Published By:

Blue Eyes Intelligence Engineering

\& Sciences Publication

(C) Copyright: All rights reserved.

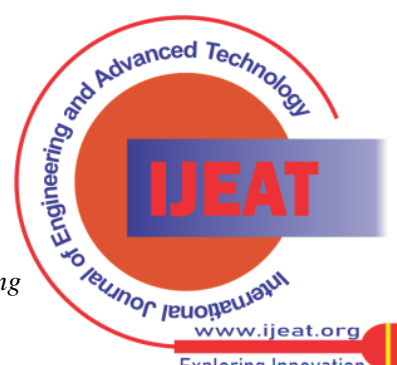

Exploring Innovation 


$$
\begin{gathered}
x_{0}, y_{0} \sum_{i=1}^{n} W_{i} \cdot\left(\rho_{i}-x_{0} \cdot \cos \left(\theta_{i}\right)-y_{0} \cdot \sin \left(\theta_{i}\right)\right)^{2}, \text { (8) } \\
\text { where }^{W_{i}}=v_{i} / V, v_{i} \text { is the total number of voting in the }
\end{gathered}
$$

parametric quantity spae for a line set by $\left(\rho_{i}, \theta_{i}\right)$ and $V$ is the total number of voting. When an appearance is estimated, the straight lines that are for away from the vanishing point more than a threshold value are suppressed. Estimated vanishing point (appearance) is shown in Figure 3(a).

\section{B. Depth Estimation}

The objective of this step is to finding the depth level of lines shows in the scene. Theoretically, the depth of a physical point related with the camera (camera setup shown in Figure. 2 ) is connected to its moving vertical position in the image or video sequence, as follows [22]

$$
z=h_{\text {cam }} \tan \left(\tan ^{-1}\left(\left(y_{s}-\frac{d}{2}\right) / f\right)+\alpha_{c a m}\right),
$$

where $y_{s}$ plays the place of the object on the sensor, $d$ is the height of the sensor, $f$ is the focal length, $Z$ the projected distance of the object on the ground to the camera, $h_{\text {cam }}$ is the camera height and $\alpha_{\text {cam }}$ is the orientation of the camera. An $\alpha_{\text {cam }} 90^{\circ}$ means that the camera position is parallel to the ground and $0^{0}$ means that bird's eye view of camera. The first step consists in finding $\alpha_{\text {cam }}$ using a least square algorithm. Later on $f$ and $d$ are unknown, we assume a standard field of view and assume that $f=34 \mathrm{~mm}$ and $d=25 \mathrm{~mm}$ The height camera's location $h_{\text {cam }}$ is only a coefficient factor that looks location on ground plane $Z$ and view on sensor $y_{s}$ we fix it to $5 m$. Estimated depth lines are shown in Figure 3(b).

\section{DETECTION AND TRACKING OF LANE CROSSING VEHICLES}

After the lane region detection, the next step is tracking of every vehicle in order to detect illegal lane crossing vehicle which is carried in online. While tracking every vehicle, we observe the tracking window and vehicle is said to be an illegal lane crossing or illegal overtaking vehicle when the distance between the moving vehicle center and straight lane line is within the user-defined threshold. The work is classified into two steps, the first step is tracking of the vehicle which yields the tracking window and the second step is vehicles lane crossing judgment.

\section{A. Tracking of Vehicles}

Our previous work [4] for tracking of vehicles in traffic video based on model-based strategy involves two steps. In

the first step, the background is subtracted and vehicles are detected in the frame $t$ we subtract with the background image and obtained prospect foreground objects represented as foreground mask. The background subtraction reduces computation time and removes the complex background. After getting the foreground mask of prospect foreground objects in frame $t$ moving vehicles are detected using Co-HOG descriptors. In the second step, the vehicle model is constructed for each vehicle in frame $t$ based on shape and texture features extracted from the foreground mask of the vehicle region using Co-HOG and CS-LBP operator. The vehicle model captures the variation in the scale of the vehicle, pose of the vehicle, and complex vehicles occlusion. After created the vehicle model for the current frame, the moving vehicle features are extracted from the vehicle image and then the vehicle model is updated. Finally, moving vehicles are tracked using the similarity measure between current frame moving vehicles and constructed vehicle model. Vehicle position is set by integrating all corresponding in the moving vehicles and vehicle model.

\section{B. Vehicle Lane Crossing Judgement}

The illegal lane crossing vehicles are detected while tracking vehicles based on vehicles tracking window location [3]. First, calculate the position of the moving vehicle center $(x, y)$.

$$
\begin{aligned}
& x=\frac{u p+d o w n}{2}, \\
& y=\frac{l e f t+r i g h t}{2},
\end{aligned}
$$

where $u p$ and down are parameters represent the location of tracking window's top and bottom boundaries. Similarly, left and right parameters are the location of tracking window's right and left boundaries.

After finding the location of vehicle center, next step is to compute the minimum distance (md) between the center of the target moving vehicle $(x, y)$ and straight lane line. In order to detect illegal lane crossing vehicle, we compute the distance between the $1 / 2$ width of the moving vehicle and minimum distance (md).

$$
\operatorname{Distance}(D)=[m d-\text { width } / 2] \text {, }
$$

where width/2 of the vehicle is defined as:

$$
\text { width } / 2=\frac{\text { right }- \text { left }}{2} \text {. }
$$

If the Distance (D) is less than user-defined threshold, the vehicle is detected as illegal lane crossing vehicle. The user defined threshold is set empirically.

After vehicles lane crossing judgment, illegal lane crossing vehicles are tracked to estimate the same target timely,

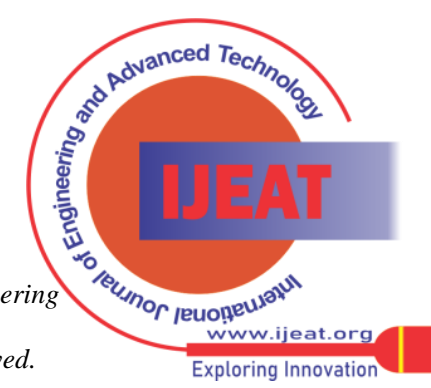


which help to predict the location of the illegal moving vehicle in a time period.

\section{EXPERIMENTAL RESULTS}

The performance evaluation of the proposed method for illegal lane crossing vehicle detection and tracking is a frame-by-frame evaluation process.

It is observed that there is no traffic video set available which contains illegal lane crossing events. Hence, we selected two video sequences which contain illegal lane crossing event from publicly available traffic surveillance video dataset such as GRAM-Road Traffic Monitoring (GRAM-RTM) [5]. In addition to publicly available video sequences, we used traffic video sequences available on the Internet and these video sequences contain illegal lane crossing events. In order to measure the performance of our proposed approach, we used evaluation metrics such as precision, recall, and f-measure. The evaluations parametric are determined as follows:

$$
\begin{gathered}
\text { recall }=\frac{\sum_{t} T P_{t}}{\sum_{t} N_{t}}, \\
\text { precision }=\frac{\sum_{t} T P_{t}}{\sum_{t}\left(T P_{t}+F P_{t}\right)}, \\
f-\text { measure }=\frac{2 * \text { recall } * \text { precision }}{\text { recall }+ \text { precision }},
\end{gathered}
$$

where $T P_{t}$ is the number of true positives (real lane crossing vehicle) at time $t, F P_{t}$ is the number of false positive (vehicle is not related with a real lane crossing vehicle) at time $t$, and $N_{t}$ is the total number of lane crossing vehicles at time $t$. Each lane crossing vehicle detected that is related with a real lane crossing vehicle is considered to be a true positive $(T P)$. For each time $t$, each detected lane crossing vehicle and real lane crossing vehicle is said to be related when both are in the same lane or area (or partially in the same area if one or two of them overlap two areas). If the lane crossing vehicle is not related with a real lane crossing vehicle, then it is considered to be a false positive $(F P)$

\section{A. Experiments on GRAM-RTM Video Subset}

The selected two video sequences from GRAM-RTM dataset are M30 and M 30 HD. These two video sequences contain illegal lane crossing vehicles in the urban highway. The selection of video sequences is based on the occurrence of illegal lane crossing event where vehicles are crossing the broken or continuous lane. The selected video sequences are having 7529 frames (M30) with the resolution $800 \times 480$ and 9390 frames (M30 HD) with resolution $1200 \times 720$ respectively. In order to generate ground truth for each video sequence, illegal lane crossing vehicles are manually detected and marked using rectangular window. The TP and FP are computed based on the comparison between results of our proposed approach with ground truth based on overlapping between tracking window and manually marked window. Table 1 presents recall, precision, and f-measure obtained for our approach on two video sequences of GRAM-RTM dataset. From the evaluation results obtained on video sequences, it is noticed that our approach yields the highest precision and recall for detecting illegal lane crossing vehicles in high roadways. The highest accuracy of our proposed approach is due to the fact that our approach uses model-based tracking of detected

vehicle under variations such as pose, lighting, and background. The existing methods performance degrades when the vehicle pose changes while vehicle crossing the lane. However, our approach gives the highest accuracy even though vehicles pose changes.

Figure 4 and Figure 5 shows the visual result of our proposed approach on M30 and M30 HD video sequences. It is observed that our approach accurately detects illegal lane crossing vehicles under various situations like lighting variations, background changes, and other factors.

Figure 6 shows false detection of our proposed approach on video sequences of GRAM-RTM. There exists some wrong detection and tracking as shown in Figure 6. These false detections are shown as green color tracking window. Because of camera's view angle, the vehicle moving is also close to the straight lane line and border line, the moving vehicle looks like partially occluded with straight lane line and borderline that makes wrong judgments.

\section{B. Experiments on Publically Available Traffic Video Sequences}

In this section, we present the experimental results of our approach for illegal lane crossing vehicles detection and tracking using publicly available video sequences. These video sequences are available on the Internet which contains illegal lane crossing events. These videos sequence have been capture on highways with various vehicle traffic and illumination conditions. We selected five videos ( $396 \times 336$ pixels, for a total duration of approximately 8 hours) that are same to traffic video surveillance. For these videos sequence, the ground truths have been manually obtained. Table 2 presents the recall and precision obtained using our approach on publicly available video sequences.

The Figure 7 shows illegal lane crossing result for five different publically available video sequences. The first column shows detected and tracked lane crossing vehicle shown using red color tracking window. The shadow of the vehicle may cross the lane and this can be found in the video frames shown in the first column. From the tracking results, it is observed that our approach not considered shadow crossing as illegal lane crossing. The efficacy of our approach is due to usage of model-based tracking. Remaining three columns shows the result of our approach on selected video sequences for illegal lane crossing.

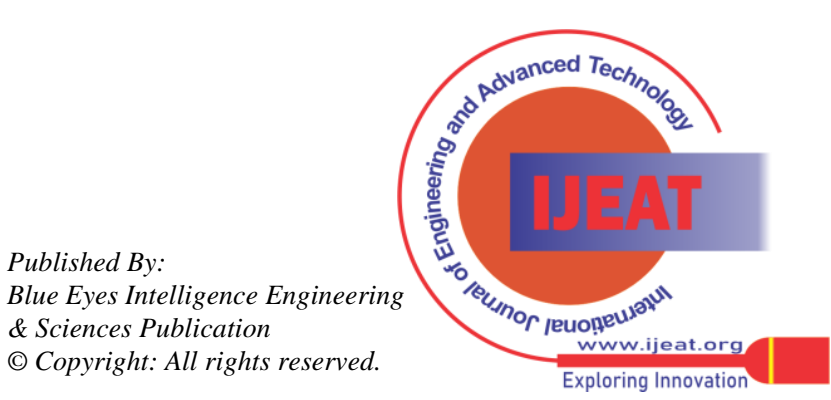


Detection and Tracking of Lane Crossing Vehicles in Traffic Video for Abnormality Analysis

Table 1. The evaluation results of our approach on selected video sequences of GRAM-RTM

\begin{tabular}{|l|c|c|c|c|}
\hline \multicolumn{1}{|c|}{ video sequence } & $\begin{array}{c}\text { Total Number of illegal } \\
\text { lane crossing vehicles }\end{array}$ & $\begin{array}{c}\text { Number of illegal lane } \\
\text { crossing vehicles detected }\end{array}$ & Recall & Precision \\
\hline M 30 & 30 & 28 & 0.93 & 0.96 \\
\hline M30 HD & 38 & 35 & 0.92 & 0.94 \\
\hline
\end{tabular}

Table 2. The evaluation results of our approach on publicly available traffic video sequences

\begin{tabular}{|c|c|c|c|c|}
\hline video sequence & $\begin{array}{c}\text { Total Number of illegal } \\
\text { lane crossing vehicles }\end{array}$ & $\begin{array}{c}\text { Number of illegal lane } \\
\text { crossing vehicles detected }\end{array}$ & Recall & Precision \\
\hline Video1 & 28 & 29 & 0.96 & 0.93 \\
\hline Video2 & 32 & 30 & 0.90 & 0.94 \\
\hline Video3 & 40 & 36 & 0.90 & 0.92 \\
\hline Video4 & 31 & 28 & 0.90 & 0.93 \\
\hline Video5 & 34 & 33 & 0.91 \\
\hline
\end{tabular}

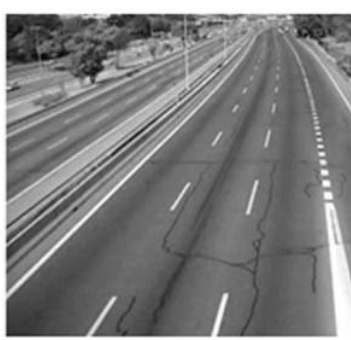

$a$

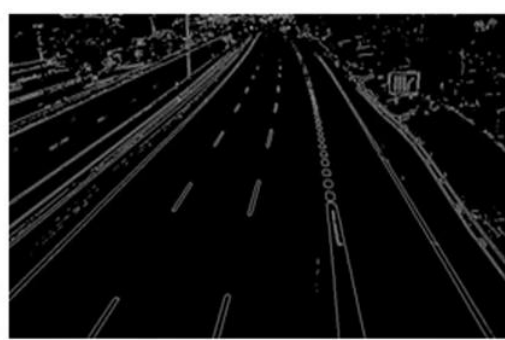

$b$

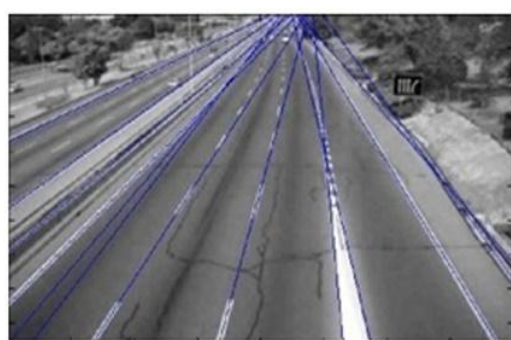

c

Figure 1. Intermediate results of detection of road region: (a) Background obtained through GMM (b) Result of Canny edge detector (c) Straight lines obtained using Standard Hough Transform

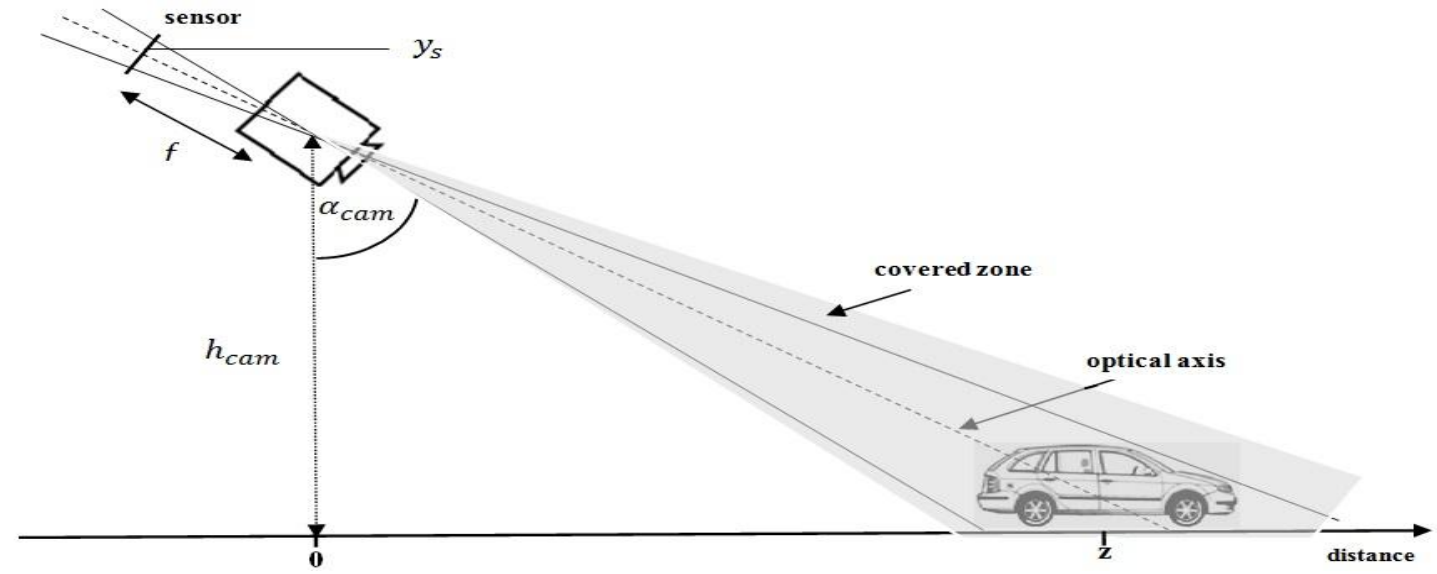

Figure 2. Camera setup

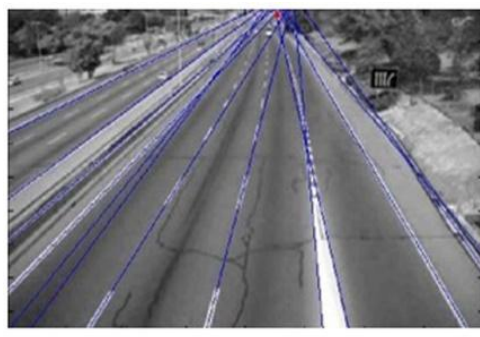

$a$

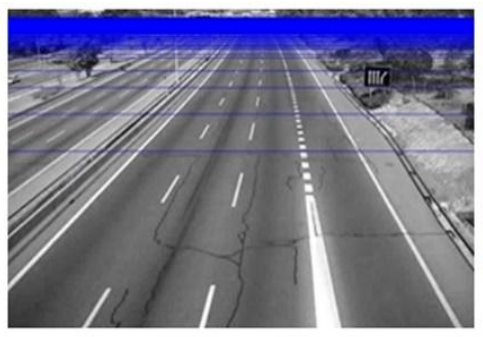

$b$

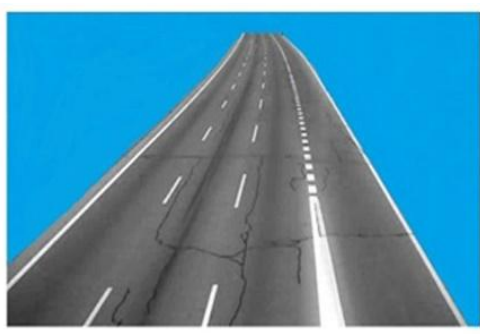

Figure 3. Result of road region detection: (a) estimated vanishing point (b) Depth estimation (c) Detected road region

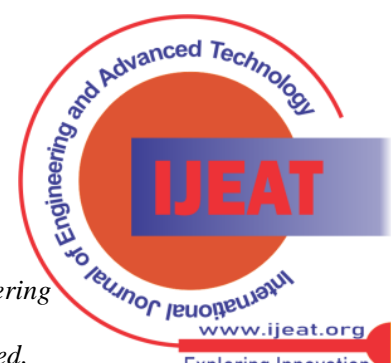
Exploring Innovation 

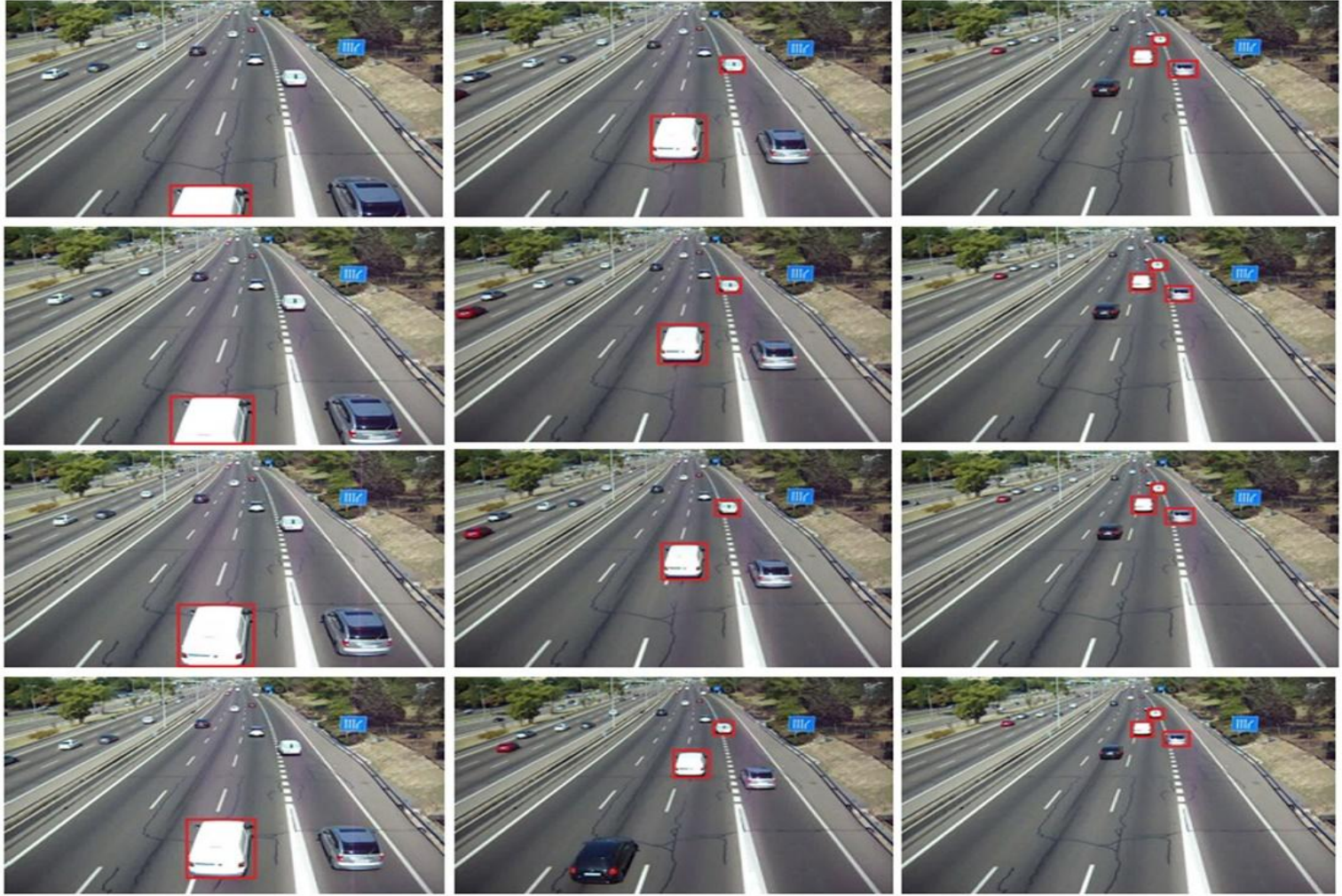

a

$\boldsymbol{b}$

\section{$c$}

Figure 4. Results of illegal lane crossing vehicles detection and tracking at different times on M30 video sequence: (a) One vehicle crossing the lane illegally (b)Two vehicles are detected as illegal lane crossing vehicles and (c) More than two vehicles are crossing the lane illegally
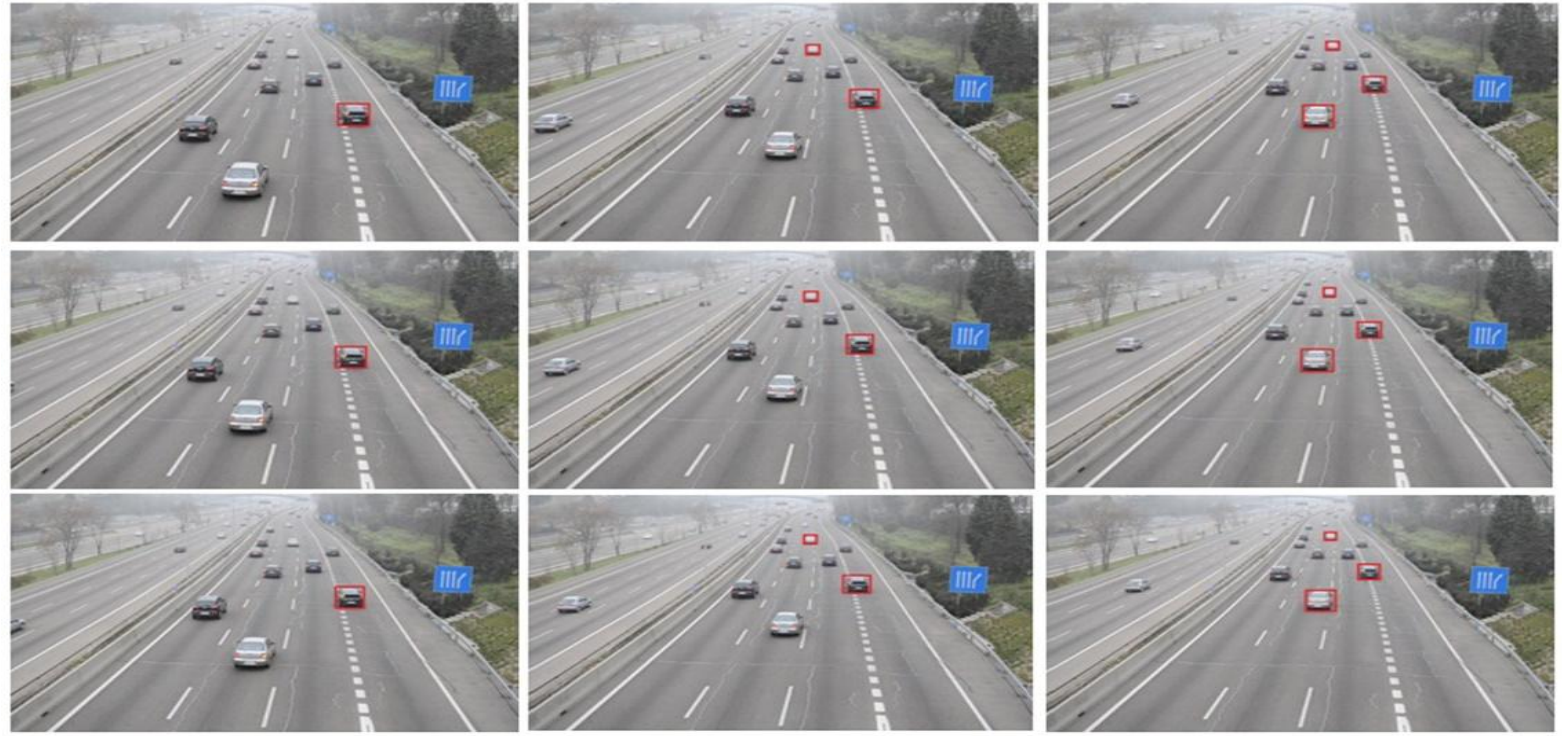

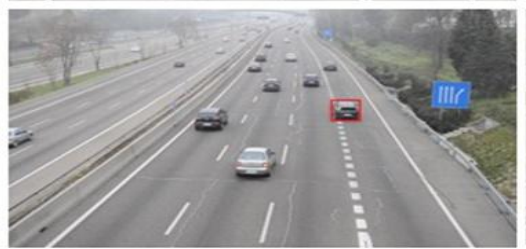

a

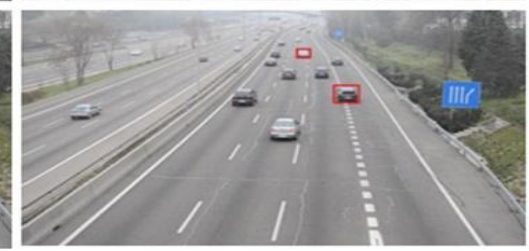

b

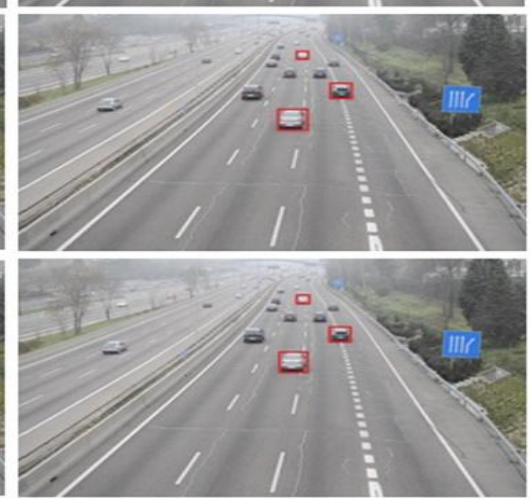

C

Figure 5. Results of illegal lane crossing vehicles detection and tracking at different times on M30 HD video sequence: (a) one illegal lane crossing vehicle, (b) a second illegal lane crossing vehicle coming out, and (c) three illegal lane crossing vehicles.

Published By:

Blue Eyes Intelligence Engineering

\& Sciences Publication

(C) Copyright: All rights reserved.

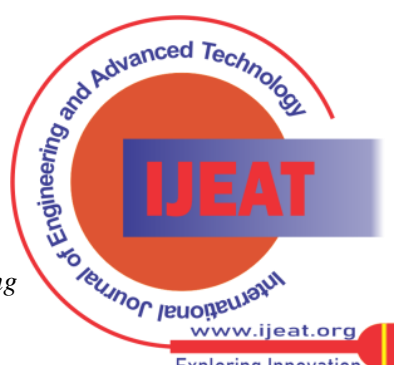




\section{Detection and Tracking of Lane Crossing Vehicles in Traffic Video for Abnormality Analysis}
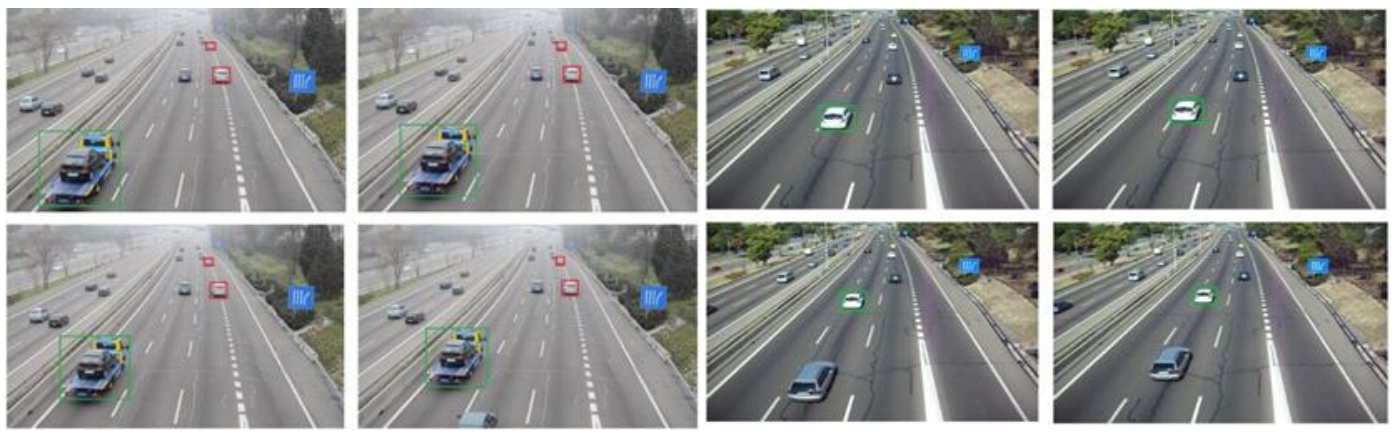

Figure 6. Some false detection results

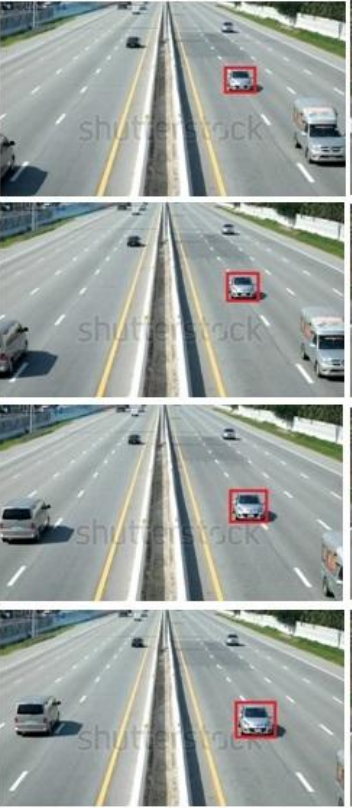

a

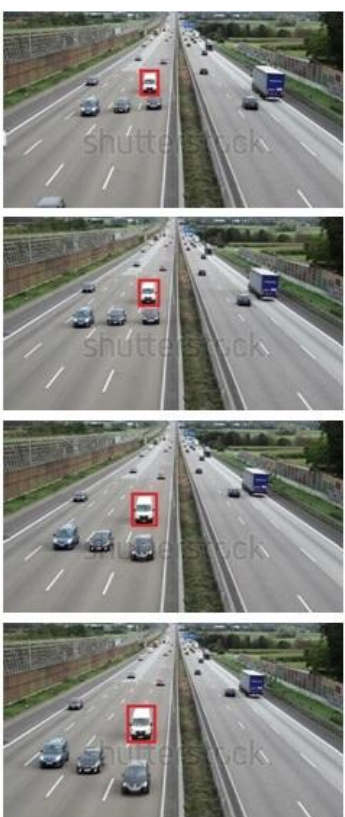

b

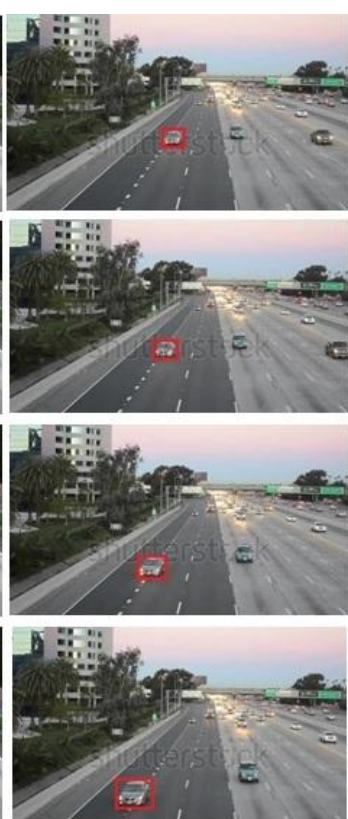

C

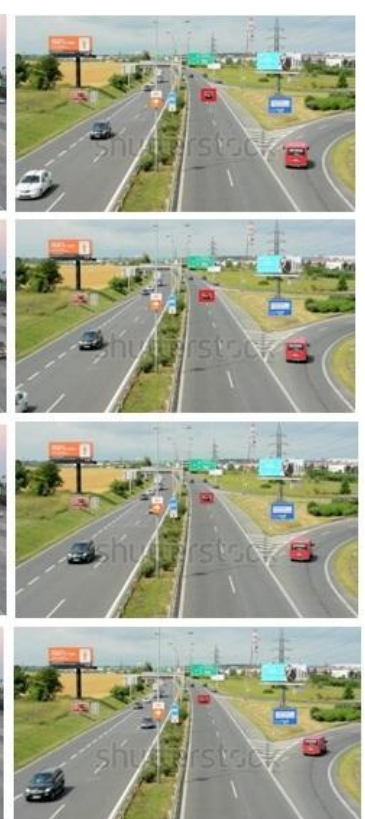

d

Figure 7. Visual results of our approach for illegal lane crossing vehicles detection and tracking at different times on publicly available traffic video sequences.

\section{CONCLUSION}

In this paper, we presented a novel approach for the detection and tracking of illegal lane crossing vehicles on urban highways. Our approach uses GMM method for background detection and the boundary of the lane is located using a Hough transformation and perspective projection technique. After the lane region detection, the next step is tracking of every vehicle in order to detect illegal lane crossing allowing to the distance between detected vehicle center and lane lines. The illegal vehicles are tracked efficiently using our previous work which uses model-based tracking technique. The evaluation results are obtained on the subset of GRAM-RTM dataset and publically available video sequences. The observational result evidence that our approach achieves the highest accuracy. The drawback of our approach is that when the vehicle is moving too close to the lane line and borderline, the vehicle looks like partially occluded with lane line and borderline that causes wrong decision and it reduces the accuracy of our approach.

\section{REFERENCES}

1. Zhang Jun, Tao Gao. \& Zheng-guang Liu, (2009) "Traffic video based cross road violation detection" In Measuring Technology and
Mechatronics Automation, International Conference,Vol. 3, pp 645-648.

2. Marikhu Ramesh, Jednipat Moonrinta, Mongkol Ekpanyapong, Matthew Dailey. \& Supakorn Siddhichai, (2013) "Police Eyes: Real world automated detection of traffic violations" In Electrical Engineering/Electronics, Computer, Telecommunications and Information Technology 10th International Conference, pp 1-6.

3. Gao Tao, Zhengguang Liu, Shiguo Lian, Shihong Yue. \& Jun Zhang, (2011) "Crossing road monitoring system based on adaptive decision for illegal situation" Applied soft computing Vol. 11, No. 7, pp 4399-4412.

4. Arun Kumar H.D. \& Prabhakar C.J., (2016) "Model Based Technique for Vehicle Tracking in Traffic Video using Spatial Local Features", Machine Learning and Applications: An International Journal, Vol. 3, No. 4, pp 1-17.

5. Guerrero-Gómez-Olmedo, Ricardo, Roberto J. López-Sastre, Saturnino Maldonado-Bascón. \& Antonio Fernández-Caballero, (2013) "Vehicle tracking by simultaneous detection and viewpoint estimation" In International Work-Conference on the Interplay Between Natural and Artificial Computation, Springer Berlin Heidelberg, pp 306-316.

6. Fu Zhouyu, Weiming Hu. \& Tieniu Tan, (2005) "Similarity based vehicle trajectory clustering and anomaly detection" In Image Processing, ICIP. IEEE International Conference, Vol. 2, pp II-602.

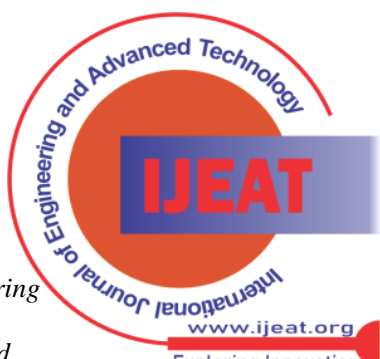
Exploring Innovation 
7. Akoz Omer. \& M Elif Karslıgil, (2010) "Video-based traffic accident analysis at intersections using partial vehicle trajectories" Signal Processing and Communications Applications Conference (SIU), 18th. IEEE, pp 499-502.

8. Tai Jen-Chao, Shung-Tsang Tseng, Ching-Po Lin. \& Kai-Tai Song, (2004) "Real time image tracking for automatic traffic monitoring and enforcement applications" Image and Vision Computing, Vol. 22(6), pp 485-501.

9. Kamijo Shunsuke, Masahiro Harada. \& Masao Sakauchi, (2004) "Incident detection based on semantic hierarchy composed of the spatio-temporal MRF model and statistical reasoning" Systems, Man and Cybernetics, IEEE International Conference. Vol. 1, pp 415-421.

10. Veeraraghavan Harini, Paul Schrater. \& Nikolaos Papanikolopoulos, (2005) "Switching kalman filter-based approach for tracking and event detection at traffic intersections" In Intelligent Control, Proceedings of the IEEE International Symposium on, Mediterrean Conference on Control and Automation, pp 1167-1172.

11. Piciarelli Claudio, Christian Micheloni. \& Gian Luca Foresti, (2008) "Trajectory-based anomalous event detection" IEEE Transactions on Circuits and Systems for video Technology Vol. 18, No.11, pp 1544-1554.

12. Hsu Wei-Lieh, Chang-Lung Tsai. \& Po-Lun Chang, (2009) "Automatic traffic monitoring method based on cellular model" In Intelligent Information Hiding and Multimedia Signal Processing, IEEE Fifth International Conference, pp 640-643.

13. Chen Lairong, Yuan Cao. \& Ronghua Ji, (2010) "Automatic incident detection algorithm based on support vector machine" In Natural Computation (ICNC), Sixth International Conference IEEE, Vol. 2, pp 864-866.

14. Dong Nan, Zhen Jia, Jie Shao, Ziyou Xiong, Zhipeng Li, Fuqiang Liu, Jianwei Zhao. \& PeiYuan Peng, (2010) "Traffic abnormality detection through directional motion behavior map" In Advanced Video and Signal Based Surveillance (AVSS), Seventh IEEE International Conference, pp 80-84.

15. Cui Lili, Kehuang Li, Jiapin Chen. \& Zhenbo Li, (2011) "Abnormal event detection in traffic video surveillance based on local features" In Image and Signal Processing (CISP), 4th International Congress, IEEE, Vol. 1, pp 362-366.

16. Babaei Peyman, (2015) "Vehicles behavior analysis for abnormality detection by multi-view monitoring" Int. Res. J. Appl. Basic Sci Vol. 9, No. 11, pp 1929-1936.

17. Piciarelli Claudio. \& Gian Luca Foresti, (2006) "On-line trajectory clustering for anomalous events detection" Pattern Recognition Letters 27, No. 15, pp 1835-1842.

18. Zivkovic Zoran, (2004) "Improved adaptive Gaussian mixture model for background subtraction" In Pattern Recognition, Proceedings of the 17th International Conference, IEEE, Vol. 2, pp 28-31.

19. Ding Lijun. \& Ardeshir Goshtasby, (2001) "On the Canny edge detector" Pattern Recognition Vol. 34, No. 3, pp 721-725.

20. Nguyen, Thuy Tuong, Xuan Dai Pham. \& Jae Wook Jeon, (2008) "An improvement of the Standard Hough Transform to detect line segments" In Industrial Technology, IEEE International Conference, pp 1-6.

21. Matessi Andrea. \& Luca Lombardi, (1999) "Vanishing point detection in the hough transform space" In European Conference on Parallel Processing, Springer Berlin Heidelberg, pp 987-994.

22. Kas Christian. \& Henri Nicolas, (2009) "Rough compressed domain camera pose estimation through object motion" In Image Processing (ICIP), 16th IEEE International Conference, pp 3481-3484.

\section{AUTHORS PROFILE}

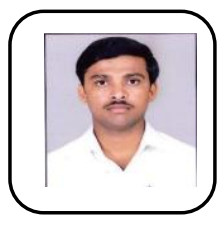

Arun Kumar H.D., Received his Ph.D. degree in Computer Science and Technology from Kuvempu University, Karnataka, India in 2017. He is currently working as Lecturer in the department of Computer Science and MCA, Kuvempu University, Karnataka, India. His research interests are image and video processing, Computer Vision and Machine Vision Short Biography

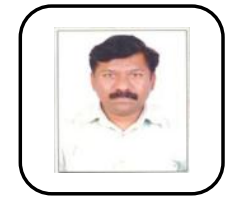

Prabhakar C.J., Received his Ph.D. degree in Computer Science and Technology from Gulbarga University, Gulbarga, Karnataka, India in 2009. He is currently working as Associate Professor in the department of Computer Science and M.C.A, Kuvempu University, Karnataka, India. His research interests are

Published By:

Blue Eyes Intelligence Engineering

\& Sciences Publication

(C) Copyright: All rights reserved.

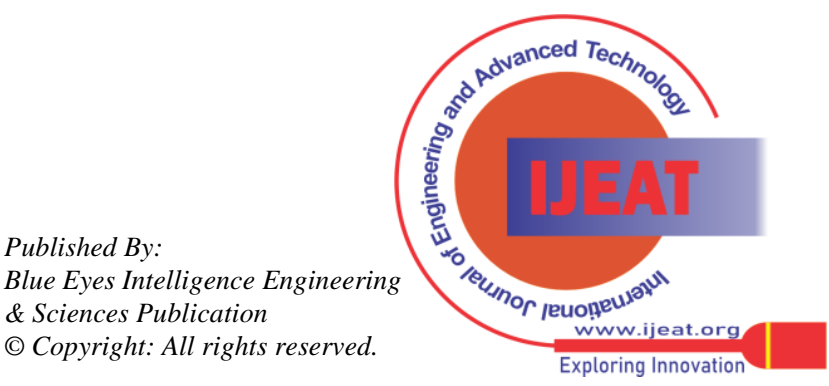

